\title{
GaN/air gap based micro-opto-electro-mechanical (MOEM) Fabry-Pérot filters
}

\author{
E. Cho ${ }^{*}, 1,2$, D. Pavlidis ${ }^{* *}, 1,2$, and E. Sillero ${ }^{3}$ \\ ${ }^{1}$ Department of High Frequency Electronics, Technische Universität Darmstadt, Merckstrasse 25, \\ 64283 Darmstadt, Germany \\ 2 Department of Electrical Engineering and Computer Science, The University of Michigan, Ann Arbor, \\ MI 48109-2122, USA \\ ${ }^{3}$ Instituto de Sistemas Optoelectrónicos y Microtecnología and Department of Electronic Engineering, \\ Universidad Politécnica de Madrid, 28040 Madrid, Spain
}

Received 27 September 2006, revised 2 November 2006, accepted 10 November 2006

Published online 31 May 2007

PACS 42.79.Ci, 42.79.Fm, 78.66.Fd, 81.15.Gh, 81.15.Hi

Structural and optical properties of Fabry-Pérot filters (FPFs) with GaN/air gap based distributed Bragg reflectors (DBRs) were studied. Reflectance of GaN/air gap DBRs on sapphire substrate was calculated from the standard transmission matrix method and results showed that $98 \%$ reflectance is achievable with only 3.5 pairs at a center wavelength of $450 \mathrm{~nm}$. The thickness of the GaN layer and the first AIN layer was determined according to the deformation induced by the residual stress. In-plane strain corresponding to growth conditions and the thickness of the $\mathrm{GaN}$ epilayer was considered for this analysis. Optical tuning efficiency and spectral range were found to be 0.27 and $25 \mathrm{~nm}$ respectively for FPFs with GaN/air gap $\left(322 \mathrm{~nm} / 113 \mathrm{~nm}\right.$ ) based DBRs and a $\lambda_{0} / 2$ air resonant cavity. The calculated pull-in voltage was $1.5 \mathrm{~V}$. Crack free AlN grown on GaN by in-house MOCVD showed an etching rate of $0.2 \mathrm{~nm} / \mathrm{min}$.

(C) 2007 WILEY-VCH Verlag GmbH \& Co. KGaA, Weinheim

1 Introduction Distributed Bragg reflectors (DBRs) are a key part in nitride optoelectronic devices such as vertical cavity surface emitting lasers, resonant cavity surface emitting diodes and resonant cavity detectors. $\mathrm{Al}_{x} \mathrm{Ga}_{1-\mathrm{x}} \mathrm{N} / \mathrm{GaN}$ or $\mathrm{Al}_{\mathrm{x}} \mathrm{Ga}_{1-\mathrm{x}} \mathrm{N} / \mathrm{AlN}$ based DBRs are mainly used because they are transparent at visible wavelengths and ultra violet wavelength ranges. Although crack free DBRs with a reflectance higher than $99 \%$ were presented at $467 \mathrm{~nm}$ and $380 \mathrm{~nm}[1,2]$, the growth of this type of DBRs is still challenging due to cracks and dislocations induced by lattice mismatch and different thermal expansion coefficients. To overcome these issues, $\mathrm{Al}_{1-\mathrm{x}} \mathrm{In}_{\mathrm{x}} \mathrm{N} / \mathrm{Al}_{1-\mathrm{x}} \mathrm{Ga}_{\mathrm{x}} \mathrm{N}$ based DBRs have been investigated. The advantage of this material system lies in its lattice-matched structure. A 40 period $\mathrm{Al}_{0.83} \mathrm{In}_{0.17} \mathrm{~N} / \mathrm{GaN}$ DBR and a 35 period $\mathrm{Al}_{0.85} \mathrm{In}_{0.15} \mathrm{~N} / \mathrm{Al}_{0.2} \mathrm{Ga}_{0.8} \mathrm{~N}$ DBR were reported recently at $450 \mathrm{~nm}$ and $343 \mathrm{~nm}$, respectively $[3,4]$. These DBRs are comparable to $\mathrm{Al}_{x} \mathrm{Ga}_{1-\mathrm{x}} \mathrm{N} / \mathrm{GaN}$ or $\mathrm{Al}_{\mathrm{x}} \mathrm{Ga}_{1-\mathrm{x}} \mathrm{N} / \mathrm{AlN}$ based DBRs mentioned above, but Indium segregation is a problem in this material system. GaN/air gap based DBRs are considered in this work to overcome drawbacks of nitride semiconductor DBRs. Air gaps can, for example, be formed by wet etching of a sacrificial layer. Dry etching can also be used as shown in several papers. Steckl presented horizontally stacked GaN gratings fabricated by focused ion beam (FIB) micromilling [5]. In most of cases, wet etching of nitride materials is assisted by UV light since nitride semiconductors are very robust to conventional chemical treatment. The corresponding method is called photoelectrochemical etching (PEC). A three-period GaN based air gap DBR was reported using bandgap-selective PEC etching with an InGaN sacrificial layer [6]. Although selective etching of nitrides is

\footnotetext{
* Corresponding authors: e-mail: cho@hf.tu-darmstadt.de; Phone: +49 6151 16 4162, Fax: +49 6151164367

** e-mail: pavlidis@hf.tu-darmstadt.de
} 
extremely difficult and rough surfaces resulting from etching are a problem, air gap based DBRs are very attractive because only 3.5 periods are needed for $98 \%$ reflectance as shown in Fig. 1a. In addition, Fabry-Pérot filters (FPFs) consisting of two GaN/air gap based DBRs can tune the resonant wavelength by controlling the air cavity length electrostatically or thermally. Micro-opto-electro-mechanical (MOEM) tunable FPFs have been reported with conventional III-V materials [7, 8]. These devices target the application of optical communication and IR gas spectroscopy systems. Similar spectroscopy applications are expected for $\mathrm{GaN}$ based FPFs because some organic materials and chemicals are sensitive to $\mathrm{UV}$ and/or to the visible region.

In this study, AlN was chosen as a sacrificial layer because it can be etched away by wet etching without additional light. Since selective etching between $\mathrm{Al}_{\mathrm{x}} \mathrm{Ga}_{1-\mathrm{x}} \mathrm{N}$ and $\mathrm{AlN}$ is possible with small amounts of $\mathrm{Al}$ content, it is feasible to realize $\mathrm{Al}_{\mathrm{x}} \mathrm{Ga}_{1-\mathrm{x}} \mathrm{N} / \mathrm{air}$ gap DBRs for wavelengths below the GaN absorption edge. To determine design parameters of GaN/air gap DBRs, strain relaxation was considered for the residual stress analysis. The optical and mechanical simulation results of the DBRs and FPFs with these DBRs will be discussed together with experimental investigations of growth and etching of AlN on sapphire.

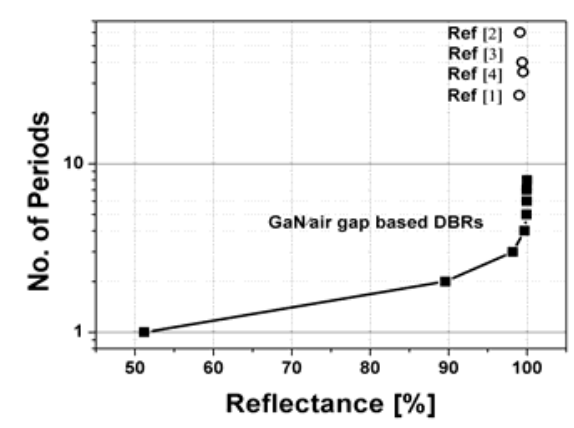

a)

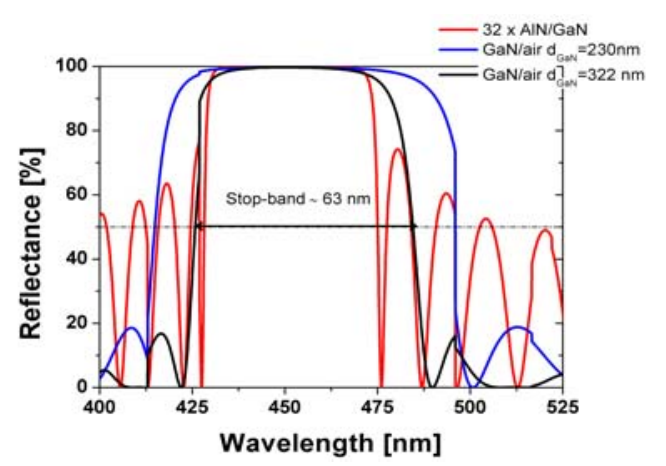

b)

Fig. 1 a) Number of GaN/air gap quarter-wave pairs vs. reflectance, b) simulated reflectance spectra of two 3.5period GaN/air gap based DBRs having different thickness and a 32-period GaN/AIN (46 nm/54 nm) DBRs.

2 Designs and results The DBR designs presented in this work employ thick AIN as the first layer on a sapphire substrate so that cracks in the following layers can be prevented [9]. The thickness of this first AlN grown layer should be optimised in consideration of the deformation of the GaN epilayer after etching. The thickness of the GaN layer and the sacrificial AIN layer correspond to $i^{*} \lambda_{0} / 4 n_{1}$ and $j * \lambda_{0} / 4 n_{2}$ for $\lambda_{0}=450 \mathrm{~nm}$ where $n_{1}$ and $n_{2}$ are the refractive indices of GaN and air respectively. $i$ and $j$ are odd integers and they are chosen according to the optical and mechanical performance of the DBRs. Reflectance simulations were performed by using the standard transmission matrix method [10]. The GaN absorption edge is below $400 \mathrm{~nm}$ and absorption was therefore excluded in this simulation. The refractive index data for GaN were taken from Brunner [11] and the value of 1.78 was used for sapphire [12]. The incident light was assumed to be normal to the surface and the incident medium was taken to be air. The reflectance spectra of two GaN/air gap DBRs with different thickness and a 32-period GaN/AIN DBR are shown in Fig. 1b. In all cases, the air gap was fixed to $113 \mathrm{~nm}$ and 3.5 periods were used. Regardless of GaN thickness, the maximum reflectance remained above $98 \%$ but the stop-band was reduced to $63 \mathrm{~nm}$ as the thickness of GaN was increased from $230 \mathrm{~nm}$ to $322 \mathrm{~nm}$. However, this value is still broader than that of a 32-period GaN/AIN DBR.

Simple square geometry with four beams was used for Fabry-Pérot filters with 3.5-period GaN/air gap based DBRs. The length of the air resonant cavity (L) is about a $\lambda_{0} / 2$. SiO ${ }_{2}$ should be deposited on the backside of the sapphire substrate as anti-reflection coating. Four different designs were considered. The design parameters are summarized in Table 1. 
Table 1 Various designs of FPFs' membrane (unit: $\mu \mathrm{m}$ )

\begin{tabular}{lcccc}
\hline & A $^{*}$ & $\mathrm{~B}^{*}$ & $\mathrm{C}^{*}$ & $\mathrm{D}^{* *}$ \\
\hline \hline Membrane width, W & 20 & 20 & 30 & 20 \\
Beam width, BW & 10 & 10 & 15 & 10 \\
Beam length, BL & 30 & 30 & 45 & 30 \\
GaN thickness, d & 0.23 & 0.32 & 0.32 & 0.32 \\
\hline
\end{tabular}

* Strain relaxation data under Ga (sub)monolayer MBE growth condition [15].

** Strain relaxation data under Ga bilayer MBE growth condition [15].

Firstly, in-plane residual stress $\left(\sigma_{\mathrm{a}}\right)$ was estimated roughly according to the relationship given in [13]. For the calculation of bi-axial stress, two sets of plastic strain relaxation data of GaN on AlN epilayer were taken from Bellet-Amalric [14]. Bottom $20 \mathrm{~nm}$ thick GaN was assumed to be removed during wet etching. The following values were used for elastic constants of GaN: $C_{11}=390 \mathrm{GPa}, C_{12}=145 \mathrm{GPa}, C_{13}$ $=106 \mathrm{GPa}, C_{33}=398 \mathrm{GPa}$ [15]. Secondly, the deformation of GaN membranes of each design was simulated by finite element method (FEM) with the estimated in-plane residual stress as above. Results are shown in Fig. 2a. The smallest deformation of the $1^{\text {st }} \mathrm{GaN}$ membrane was about $0.8 \mu \mathrm{m}$ for design $\mathrm{B}$ while design $\mathrm{D}$, with the same geometry as design $\mathrm{B}$, was deflected as much as $1.1 \mu \mathrm{m}$. This difference is due to the different strain relaxation mechanism corresponding to growth conditions. GaN epilayer grown under Ga bi-axial growth condition (design D) contains higher gradient relaxation above $20 \mathrm{~nm}$ thickness than under Ga (sub)monolayer growth condition (design B) and it leads to larger deformation of the membrane. This result demonstrates that growth conditions are a very important factor together with membrane geometry in design optimization. It is worth to note that stress in epilayers grown by MOCVD is very different from that present in MBE grown layers. Since relaxation data of GaN on AIN epilayer as a function of thickness are not available from MOCVD growth at this time, MBE data were employed in this paper. These are expected to provide a first estimate of the trends and parameter dependences applicable to the new scheme of GaN/air gap based MOEM FPFs discussed here. According to the theoretical calculation of strain relaxation in $1 \mu \mathrm{m} \mathrm{GaN}$ epilayer (with $100 \mathrm{~nm}$ AlN buffer layer) on sapphire [16] is about $1 \mathrm{GPa}$ which is close to that of design $\mathrm{B}$. The relaxation occurs more quickly in epilayers grown by MOCVD than by MBE, more gradual relaxation is expected above $20 \mathrm{~nm}$ and this would lead to less deformation of the GaN membrane; this is to be confirmed experimentally in the future with our in-house MOCVD [17]. Deformation of consequent GaN membranes was $25 \%$ less than that of the $1^{\text {st }} \mathrm{GaN}$ membrane based on the layer by layer analysis [18]. The differences of deformation between adjacent membranes are in the range of 2 to $15 \mathrm{~nm}$.

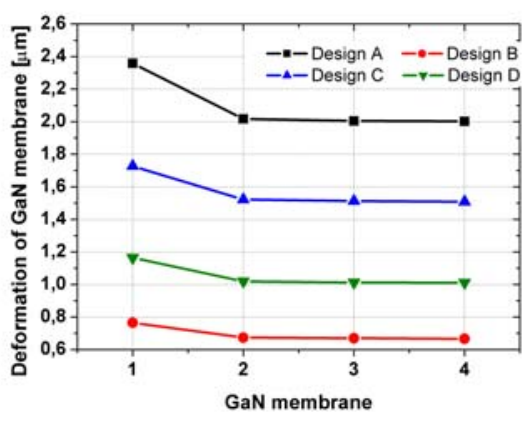

a)

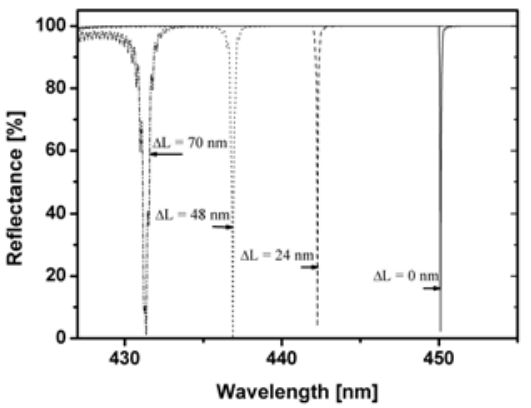

b)

Fig. 2 a) The deformation of GaN membranes for different FPFs' designs. GaN layers from the bottom to the top were labeled from 1 to 4 . b) Reflectance spectra of FPFs corresponding to air resonant cavity lengths. 
The reflectance spectra of FPFs with design B corresponding to air resonant cavity lengths are shown in Fig. 2b. By controlling the resonant cavity length $(\Delta \mathrm{L})$ up to $70 \mathrm{~nm}$, the peak wavelength shifted from $450 \mathrm{~nm}$ to $431.4 \mathrm{~nm}$ and FWHM is $0.04 \mathrm{~nm}$ and $0.42 \mathrm{~nm}$, respectively. The optical tuning efficiency of $\Delta \lambda / \Delta \mathrm{L}$ is about 0.27 and a pull-in voltage of $1.5 \mathrm{~V}$ was calculated.

Experimental investigation of the proposed concepts necessitates the growth of AlN layers which can be selectively etched with respect to GaN. AIN layers were grown for this purpose with our in-house lowpressure Organometallic Vapor Phase Epitaxy (LP-OMVPE). The TMAl flow rate varied from 7-67 $\mu \mathrm{mol} / \mathrm{min}$ and ammonia flow rate varied from $20-70 \mathrm{mmol} / \mathrm{min}$. Growth pressure was kept below 50 torr. A $0.5-1 \mu \mathrm{m}$ thick AIN layer was grown between 1040 and $1080{ }^{\circ} \mathrm{C}$ after an about $10 \mathrm{~nm}$ thick low temperature (LT) AIN nucleation layer grown at $700-900^{\circ} \mathrm{C}$. The typical growth rate was $1 \mu \mathrm{m} / \mathrm{hr}$ as determined by in-situ laser reflectance monitoring. Etching was attempted with a $4: 1 \mathrm{H}_{2} \mathrm{O} / \mathrm{AZ} 400 \mathrm{~K}$ solution and an etching rate of $0.2 \mathrm{~nm} / \mathrm{min}$ was measured.

3 Conclusion Designs of GaN/air gap based DBRs and Fabry-Pérot filters were proposed at the center wavelength $450 \mathrm{~nm}$ based on strain relaxation data of MBE growth. FEM analysis indicates that growth condition is an important factor together with membrane geometry. As the result, the thickness of $\mathrm{GaN}$ and air gap was determined as $322 \mathrm{~nm}$ and $113 \mathrm{~nm}$, respectively. Simulation results of FPFs with the proposed design showed that optical tuning efficiency was about 0.27 and spectral range was $25 \mathrm{~nm}$. A pull-in-voltage of about $1.5 \mathrm{~V}$ was calculated with $\Delta \mathrm{L}=70 \mathrm{~nm}$. This design should be adjusted in case of MOCVD grown layers by considering the corresponding strain relaxation data. Absorption of a GaN epilayer due to doping and deformation of each layer based on multiplayered heteroepitaxial structures must also be taken into account for further design improvement. AIN epilayers were grown by in-house MOCVD to evaluate of the feasibility of GaN/air gap DBRs and showed an etching rate of $0.2 \mathrm{~nm} / \mathrm{min}$.

Acknowledgements The authors acknowledge the support from Deutsche Forschungsgemeinschaft and Graduiertenkolleg on Tunable Integrated Components in Microwave Technology and Optics (TICMO).

\section{References}

[1] H. M. Ng, T. D. Moustakas, and S. N. G. Chu, Appl. Phys. Lett. 76, 2818 (2000).

[2] K. E. Waldrip, J. Han, J. J. Fiegle, H. Zhou, E. Makarona, and A. V. Nurmikko, Appl. Phys. Lett. 78, 3205 (2001).

[3] J. Dorsaz, J.-F. Carlin, S. Gradecak, and M. Ilegems, J. Appl. Phys. 97, 84505 (2005).

[4] E. Feltin et al., Appl. Phys. Lett. 88, 051108 (2006).

[5] A. J. Steckl and I. Chyr, J. Vac. Sci. Technol. B 17, 362 (1999).

[6] R. Sharma, E. D. Haberer, C. Meier. E. L. Hu, and S. Nakamura, Appl. Phys. Lett. 87, 051107 (2005).

[7] M. Strassner, J. C. Esnault, L. Leroy, J.-L. Leclercq, M. Carrigues, and I. Sagnes, IEEE Photon. Technol. Lett. 17, 804 (2005).

[8] P. Bondavalli, T. Benyattou, M. Garrigues, J. L. Leclercq, S. Jourba, C. Pautet, and X. Hugon, Sens. Actuators A 94, 136 (2005).

[9] A. Bhattacharyya, S. Iyer, E. Iliopoulos, A. V. Sampath, J. Cabalu, T. D. Moustakas, and I. Friel, J. Vac. Sci. Technol. B 20, 1229 (2002).

[10] H. A. Macled, Thin Film Optical Filters, 2nd ed. (McGraw-Hill, New York, 1989).

[11] D. Brunner, H. Angerer, E. Bustarret, F. Freudenberg, R. Höpler, R. Dimitrov, O. Ambacher, and M. Stutzmann, J. Appl. Phys. 82, 5090 (1997).

[12] M. Shubert, T. E. Tiwald, and C. M. Herzinger, Phys. Rev. B 61, 8187 (2000).

[13] V. S. Harutyunyan, A. P. Aivazyan, E. R. Weber, Y. Kim, Y. Park, and S. G. Suburamanya, J. Phys. D, Appl. Phys. 34, A37 (2001).

[14] E. Bellet-Amalric, C. Adelmann, E. Sarigiannidou, J. L. Rouvière, G. Feuillet, E. Monroy, and B. Daudin, J. Appl. Phys. 95, 1127 (2004).

[15] A. Polian, M. Grimsditch, and I. Grzegory, J. Appl. Phys. 79, 3343 (1996).

[16] K. Barghout and J. Chaudhuri, J. Mater. Sci. 39, 5817 (2004).

[17] S. M. Hubbard, G. Zhao, D. Pavlidis, W. Sutton, and E. Cho, J. Cryst. Growth. 284, 297 (2005).

[18] T. L. Song, S. J. Chua, E. A. Fitzgerald, P.Chen, and S. Tripathy, Appl. Phys. Lett. 83, 1545 (2003). 